Original

\title{
Nuevas terapias de diana celular: indicaciones, resultados y tolerabilidad
}

\author{
Joaquim Bellmunt*, Antoni Gelabert-Mas** \\ *Servicio de Oncología Médica (GU y GI). **Servicio y Cátedra de Urología. Hospital del Mar. Barcelona, España
}

\section{TERAPIAS DIRIGIDAS A DIANAS TERAPÉUTICAS EN EL CCR}

Los avances en la comprensión de la biología molecular del RCC ha dado lugar al desarrollo de tratamientos biológicos dirigidos (terapias diana) que representan, por vez primera en más de quince años de tan solo disponer de las citocinas como arma terapéutica, un avance significativo en el tratamiento de esta enfermedad, con una mejora importante en la supervivencia libre de enfermedad y con algunos fármacos, de la supervivencia global. Asimismo, el perfil de toxicidad con respecto a los tratamientos considerados estándar como las citoquinas han mejorado de forma ostensible.

Se han descrito varias dianas terapéuticas de posible interés en el tratamiento del CCR. Entre otras, las dianas mejor caracterizadas para el tratamiento del CCR son las implicadas en la proliferación celular, como la familia de receptores del factor de crecimiento epidérmico (EGFR), la vía del mTOR y la vía de la Raf-kinasa, así como las implicadas en la angiogénesis, como el factor de crecimiento endotelial vascular (VEGF) y su receptor (VEGFR) y el receptor del factor de crecimiento derivado de plaquetas (PDGFR).

Destaca la inactivación por mutación o hipermetilación en el gen de von Hippel Lindau (VHL) (presente en el 75\% de los CCR esporádicos) que dan lugar a una regulación positiva de varios genes implicados en la angiogénesis, como el VEGFR y $\mathrm{PDGFR}^{1}$. En el momento actual seis estudios han demostrado una prolongación significativa de la superciencia libre de progresión, de la supervivencia global o de ambos (Tabla 1).

Aunque no todos los pacientes con CCR presentan anormalidades en el gen VHL, casi todos los pacientes evaluados muestran una sobrerregulación del RNA mensajero (mRNA) del VEGF, o niveles elevados de la proteína $\mathrm{VEGF}^{2}$, lo cual sugiere que vías alternativas independientes de $V H L$ podrían promover la expresión de VEGF, y que probablemente éstas estén implicadas en la oncogénesis del RCC. Otras vías activadas en el CCR son la vía del EGFR y la de la mTOR, que regulan la supervivencia y el crecimiento celular.

Básicamente se han estudiado dos estrategias diferentes para inhibir estas dianas: los anticuerpos monoclonales como bevacizumab, ABX-EGF, cetuximab, y las moléculas pequeñas inhibidoras de la tirosin-kinasa como sunitinib, sorafenib, pazopanib, PTK 787 y axitinib. Otro fármaco interesante es temsirolimus. Los estudios en fase II con estos fármacos han constatado una actividad clínica sustancial en el carcinoma de células renales avanzado. Se ha descrito un efecto beneficioso sobre la supervivencia global con el uso de temsirolimus, y de la supervivencia libre de progresión con sunitinib, sorafenib, la combinación bevacizumab/interferon y con everolimus, en ensayos aleatorizados que han dado lugar a la rápida autorización de sunitinib, sorafenib, bevacizumab/interferon y temsirolimus por la FDA y/o la EMEA para el CCR avanzado. No obstante, conforme se desarrollan nuevos tratamientos, aparecen nuevos desafíos relativos al uso óptimo de estos fármacos dirigidos a dianas.

\section{Inhibidores de mTor}

Diversos compuestos, tales como rapamicina, CCI-779 (temsirolimus) y RAD-001, han sido desarrollados para inhibir mTOR y prevenir de esta manera la iniciación translacional ${ }^{3,4,5}$. En el laboratorio, la inactivación de pVHL sensibiliza las células a la inhibición de mTOR de una manera dependiente de $\mathrm{HIF}^{6}$ . En estudios clínicos fase II en pacientes con CCR metastásico refractarios a citoquinas, el tratamiento con el inhibidor de mTOR CCI-779 (temsirolimus), 
produjo una tasa de respuestas objetivas del $7 \%$, incluyendo una respuesta completa y un $17 \%$ de estabilizaciones a las 24 semanas $^{7}$. La notable actividad de este fármaco en pacientes con CCR con características de mal pronóstico, propició un estudio fase III comparando CCI-779, IFN- $\alpha$ o la combinación de los dos en 626 pacientes de mal pronóstico, demostrando un aumento significativo de la supervivencia para la rama de CCI-779 (10,9 meses), comparado con IFN- $\alpha$ (7,3 meses) o la combinación de ambos $(8,4 \text { meses })^{8}$. Este es el primer estudio que demuestra un aumento significativo de la supervivencia en pacientes con CCR metastático en pacientes con criterios de mal pronóstico, lo que ha determinado su reciente aprobación por la FDA y la EMEA de forma global.

En la misma línea que temsirolimus pero por vía oral, se ha desarrollado RAD001 (everolimus) un inhibidor oral de la serina-treonina kinasa de mTOR. En un ensayo clínico fase II en 25 pacientes con CCR metastásico se demostró que RAD001 fue bien tolerado cuando se administraba por vía oral a una dosis de $10 \mathrm{mg}$ diarios sin interrupciones (ciclo de 28 días). Los resultados de eficacia pusieron de manifiesto una actividad antitumoral prometedora demostrada por una tasa de respuestas parciales del 36\% y una prolongada mediana de tiempo a la progresión de más de 3 meses en el 86\% de los pacientes $^{9}$. En un estudio fase III en pacientes con cáncer renal que habían progresado a uno mas tratamiento con inhibidores de tirosin cinasa, y randomizados a recibir everolimus o placebo, los pacientes que recibieron everolimus presentaron una mediana de supervivencia libre de progresión de 4,0 meses (95\% CI: 3,7-5,5) comparado con 1,9 con los que recibieron placebo (95\% CI: 1,8-1,9). Demostración de que los inhibidores de mTor son una opción en tratamiento al fallo de inhibidores de tirosin cinasa como sorafenib o sunitinib ${ }^{10}$.

\section{Terapias antiangiogenicas dirigidas a factores de crecimiento endotelial vascular (VEGF) o a sus receptores}

El carcinoma renal de células claras es un tumor muy vascularizado y produce una gran variedad de moléculas proangiogénicas, incluyendo el producto de los genes de respuesta a HIF como el factor de crecimiento endotelial vascular (VEFG). VEGF estimula la proliferación y la supervivencia de las células endoteliales. Además de sus efectos proangiogé- nicos, VEGF es capaz de suprimir la respuesta inmune antitumoral ${ }^{11,12}$. El receptor de VEGF (VEGFR-2 ó KDR) está sobreexpresado en las células del CCR, lo que sugiere la posibilidad de que se produzca un mecanismo de estimulación autocrino, además de los efectos paracrinos del VEGF sobre las células endoteliales ${ }^{13}$.

Diversos agentes capaces de inhibir VEGF o su receptor $\mathrm{KDR}$, muestran actividad contra CCR. En un ensayo fase II randomizado, los pacientes con carcinoma renal metastático que fueron tratados con el anticuerpo monoclonal dirigido contra VEGF, bevacizumab, mostraron un aumento significativo en el tiempo a la progresión (4,8 meses versus 2,5 meses), lo que proporciona la prueba de concepto de la eficacia de la terapia antiangiogénica ${ }^{14}$. En un ensayo recientemente publicado, se comparó la combinación de bevacizumab $(10 \mathrm{mg} / \mathrm{Kg}$ cada 2 semanas) con IFN- $\alpha$ (9 MU 3 veces por semana) versus IFN- $\alpha$ sólo en 649 pacientes con CCR avanzado, demostrando un aumento de la tasa de respuesta (31\% vs 13\%) y del tiempo a la progresión (10,2 vs 5,4 meses, HR 0,63; $\mathrm{P}^{15}$. Los resultados de un estudio similar (CALGB 90206) presentado en ASCO-GU 2008 están pendientes de ser publicados confirman el beneficio de la combinación ${ }^{16}$.

Múltiples inhibidores de la actividad tirosinkinasa del KDR (VRGFR-2), incluyendo SU11248 (sunitinib), BAY43-9006 (sorafenib) y AG-013736 (axitinib) también han demostrado una actividad significativa contra este tipo de tumor ${ }^{17-19,23}$.

SU11248 (sunitinib) es un inhibidor oral multiselectivo de la actividad tirosin-kinasa del VEGFR-2 y PDGFR- $\beta$, con actividad adicional sobre c-Kit y FLT-3. Sunitinib ha demostrado en dos estudios fase II en pacientes refractarios a citoquinas, una tasa de respuesta del 40\%, con una tasa de estabilizaciones $>3$ meses del 27\%. La mediana del tiempo a la progresión fue de 8.5 meses $^{17}$. Este ensayo dio lugar a la aprobación de este fármaco por la FDA y la EMEA. Un reciente ensayo fase III randomizado ha comparado sunitinib versus IFN- $\alpha$ como tratamiento de primera línea en 750 pacientes con RCC metastásico a la dosis de $50 \mathrm{mg} /$ día vía oral durante 4 semanas seguido de 2 semanas de descanso ${ }^{21}$. Los resultados actualizados en supervivencia de este estudio, han sido presentados en el Congreso de la American Society of Clinical Oncology de 2008 y confirman un aumento de la tasa de respuesta (46\% versus $12 \%$ ), de la mediana de supervivencia 
libre de progresión (11 meses versus 5 meses) hazard ratio 0,52 (95\% confidence interval, 0,430,$61 ; \mathrm{P}^{18}$. Los pacientes tratados con sunitinib consiguieron una mediana de supervivencia global de 26.4 meses (95\% CI: 23.0-32.9) en comparación con 21,8 meses (95\% CI: 17,9-26,9) en los que recibieron interferon (hazard ratio 0.821 [95\% CI: 0.673-1.001]; $\mathrm{P}=0.0510$ (log-rank test). La mediana de supervivencia censando los pacientes que fueron cruzados a recibir el brazo alternativo de tratamiento fue de 26,4 meses (95\% CI: 23,0-32,9) para los que recibieron sunitinib y de 20 meses (17.8-26.9) para los que recibieron interferon (hazard ratio 0,808 [95\% CI: 0,661-0,987]; P=0,0362 (log-rank test) $^{24}$. El estudio demuestra que con las nuevas dianas terapéuticas hemos doblado la expectativa de supervivencia a nuestros pacientes

Estos resultados, junto con una mejora en la calidad de vida de los pacientes tratados con sunitinib, así como un mejor perfil de toxicidad, lo que hace que sunitinib sea actualmente una opción adecuada en el tratamiento de primera línea en pacientes con CCR avanzado.

BAY43-9006 (sorafenib) es tambien un inhibidor de multiples cinasas implicadas en angiogenesis (KDR y PDGFR- $\beta)^{23}$ pero adicionalmente tiene la peculiaridad de inhibir la via de proliferación asociada a Raf. En ensayos fase I, se constató su actividad en cáncer renal y en carcinoma hepatocelular (actualmente con beneficios en la supervivencia en pacientes con en este tipo tumoral) con respecto a otros tipos de neoplasias epiteliales. Sin embargo, es posible que la inhibición de Raf por sorafenib sea relevante en este tipo de tumor desde el punto de vista tambien de la angiogenesis, ya que Raf ha sido implicado en la supervivencia de las células endoteliales $^{22}$. Un estudio fase II de randomización discontinuada (tratamiento randomizando tan solo al subgrupo de pacientes con RCC metastático que presentan estabilización de la enfermedad) confirmó el aumento en la mediana de la supervivencia libre de progresión para pacientes tratados con sorafenib. Posteriormente Un ensayo fase III randomizado (Estudio TARGET) ha comparado sorafenib a la dosis de $400 \mathrm{mg} / 12$ horas vía oral con placebo en 903 pacientes con CCR metastático que habían recibido tratamiento previo con citoquinas, demostrando una aumento significativo de la mediana de supervivencia libre de progresión (5,5 meses para pacientes tratados con sorafenib versus 2,8 meses para pacientes tratados con placebo, $\operatorname{HR} 0,44$; $\mathrm{P}<0.01)^{23}$. Con estos datos, la FDA y la EMEA aprobaron el uso de sorafenib para el tratamiento de todo tipo de pacientes con RCC metastático. La actualización de este estudio presentada en ASCO 2007 demuestra un beneficio para la supervivencia en estos pacientes cuando no se tiene en cuenta a los pacientes del grupo placebo que recibieron sorafenib al abrirse el ciego del estudio. Un pequeño estudio fase II randomizado que comparaba sorafenib versus IFN- $\alpha$ en primera línea de tratamiento en pacientes con CCR avanzado, no ha permitido demostrar beneficio en términos de supervivencia libre de progresión con respecto a interferon. El estudio no obstante, mostró un mejor perfil de toxicidad y una mejoría en la calidad de vida de los pacientes que recibían sorafenib ${ }^{25}$.

AG-013736 (axitinib) fármaco de dispensación oral, e inhibidor de múltiples receptores tirosinkinasa, (VEGFR-1, VEGFR-2, VEGFR-3, c-Kit y PDGFR- $\beta$ ) con actividad antiangiogénica. Los resultados de un estudio multicéntrico fase II con AG013736 en el CCR se presentaron en la reunión anual de la ASCO de $2005^{34}$ y han sido reportados por Rixe ${ }^{20}$. A 52 pacientes con CCR metastásico resistente a citoquinas $(87 \%$ con una histología de células claras) se les administró 5 mg de AG-013736 dos veces al día durante cuatro semanas. Los resultados mostraron una respuesta parcial en el $46 \%$ de los pacientes y una estabilización de la enfermedad en otro $40 \%$.

\section{TGF $-\alpha$ y receptor del factor de crecimiento epidérmico}

TGF- $\alpha$ es otro de los factores de crecimiento de respuesta a HIF y es mediador de una estimulación autocrina en las células de carcinoma renal mediante la activación del receptor del factor de crecimiento epidérmico (EGFR) expresado en su superficie ${ }^{26-28}$. La inhibición farmacológica de EGFR es suficiente para inhibir el crecimiento de líneas celulares de RCC inoculadas bajo la piel de ratones inmunocomprometidos $^{29,30}$. Pequeñas moléculas inhibidoras del actividad tirosin-kinasa del EGFR (erlotinib y gefitinib) y anticuerpos monoclonales (cetuximab) están actualmente aprobados para el tratamiento de otros tipos de tumores. Desafortunadamente, los inhibidores de EGFR testados como agentes únicos en RCC metastático han demostrado una actividad clínica muy limitada ${ }^{31,32}$. Por el contrario, un ensa- 
Tabla 1. Resumen de los estudios fase III con terapias dirigidas a dianas terapéuticas en el cáncer renal

\begin{tabular}{|c|c|c|}
\hline Diseño del estudio & $\begin{array}{l}\text { Linea de tratamiento/ } \\
\text { Caracteristicas de los pts }\end{array}$ & Beneficio clínico \\
\hline Sorafenib vs placebo (23) & $2^{a}$ linea / ECOG 0-1 & $\begin{array}{l}\text { Mediana PFS } 5.5 \text { vs } 2.8 \text { mo } \\
\text { Mejor SG } \\
\text { (censurando datos de crossover) }\end{array}$ \\
\hline Sunitinib vs IFN (24) & $1^{\mathrm{a}}$ linea /ECOG PS 0-1 & $\begin{array}{l}\text { Mediana PFS } 11 \text { vs } 5 \text { mo } \\
\text { Mediana SG } 26 \text { vs } 22 \text { mo* }\end{array}$ \\
\hline $\begin{array}{l}\text { Bevacizumab + IFN vs } \\
\text { placebo + IFN (15) }\end{array}$ & $1^{\mathrm{a}}$ linea & Mediana PFS 10.2 vs 5.4 mo \\
\hline $\begin{array}{l}\text { Bevacizumab + IFN vs } \\
\text { IFN (16) }\end{array}$ & $1^{\mathrm{a}}$ linea & Mediana PFS 8.5 vs 5.2 mo \\
\hline Everolimus vs placebo(10) & $2^{\mathrm{a}}$ linea post TKI & Mediana PFS 4.0 vs $1.9 \mathrm{mo}$ \\
\hline
\end{tabular}

SG: Supervivencia global, PFS: Supervivencia libre de progresión TKI: Inhibidores de tirosin cinasa

*Significativo cuando se excluyen los pacientes que recibieron tratamiento con Sunitinib al progresar a IFN

** La comparación es de Temsirolimus frente a IFN; mediana de SG en el brazo de temsirolimus + IFN fue de 8 meses

yo piloto de fase II que evaluó la combinación de la inhibición de EGFR y VEGF con erlotinib y bevacizumab, respectivamente, en RCC metastático, demostró resultados sorprendentes con una tasa de respuesta del 25\% y una tasa de estabilizaciones a las 8 semanas del $61 \%^{33}$.

Para confirmar estos hallazgos, se diseño un estudio randomizado de monoterapia con bevacizumab versus la combinación bevacizumab/erlotinib. Los resultados preliminares no demostraron ningún beneficio de la combinación versus bevacizumab como agente único ${ }^{34}$.

\section{Combinaciones de fármacos}

Como ha sido descrito anteriormente, un gran número de productos de los genes de respuesta a HIF están implicados en el desarrollo del RCC. La inhibición individual de dichas proteínas o de sus receptores ha demostrado resultados muy prometedores, como es le caso del VEGF/KDR. La inhibición combinada de vías de señalización colaterales (combinaciones horizontales,) mediante agentes que inhiben diferentes dianas protumorigénicas de HIF (como VEGF, PDGF y TGF-a) es una aproximación racional al éxito de la combinación de agentes. Por ejemplo, agentes que bloquean VEGF (bevacizumab) o VEGFR (sorafenib, sunitinib o AG-013736) pueden ser combinados con inhibidores de EGFR (erlotinib), inhibidores de mTOR (temsirolimus o everolimus) o ambos, mediante una inhibición horizontal.
Una aproximación alternativa sería combinar agentes que inhiben indirectamente HIF mediante la inhibición de los factores de crecimiento de respuesta a HIF y de sus receptores como, por ejemplo, la combinación de un inhibidor de mTOR con bevacizumab y/o un inhibidor de KDR como sunitinib o sorafenib (combinaciones verticales). En este esquema, la inhibición submáxima de cada componente en la vía de señalización puede producir un profundo efecto, ya que la inhibición global de dicha vía variará según el producto de los efectos de cada componente. Sin embargo, las combinaciones de terapias moleculares dirigidas necesitan ser evaluadas sistemáticamente, ya que la combinación de agentes puede tener efectos tóxicos aditivos sin producir necesariamente un claro beneficio clínico.

\section{CONCLUSIONES}

El gen supresor tumoral VHL está mutado o silenciado en la mayoría de los casos de carcinomas renales de células claras. La pérdida de pVHL produce la estabilización de HIF- $\alpha$ dando lugar a la transcripción de genes de respuesta a HIF. Muchos de los productos de dichos genes han demostrado ser protumorigénicos en el desarrollo del RCC. Estos avances en la comprensión de la biología molecular del RCC ha dado lugar al desarrollo de tratamientos biológicos dirigidos que representan, por vez primera en más de quince años de tan solo disponer de las citoquinas como arma terapéutica, 
un avance significativo en el tratamiento de esta enfermedad, con una mejora importante en el perfil de toxicidad con respecto a los tratamientos considerados estándar. Entre ellos, sunitinib y la combinación bevacizumab/interferon se ha impuesto como el tratamiento de primera línea de pacientes con enfermedad avanzada. Sin embargo, las nuevas terapias dirigidas contra dianas moleculares plantean nuevos desafíos e interrogantes como son: la secuencia de administración, el beneficio de la terapia combinada, la determinación de factores predictivos de respuesta, mejora del perfil de toxicidad a largo plazo, etc. Varios ensayos clínicos están actualmente en marcha usando estos agentes en combinación o en el tratamiento adyuvante tras la cirugía en pacientes de alto riesgo.

\section{REFERENCIAS}

1. Haase VH. The VHL/HIF oxygen-sensing pathway and its relevance to kidney cancer. Kidney Int. 2006;69(8):1302-1307.

2. Rini BI, Small EJ. Biology and clinical development of vascular endothelial growth factor-targeted therapy in renal cell carcinoma. J Clin Oncol. 2005;23(5):1028-1043.

3. Treins C, Giorgetti-Peraldi S, Murdaca J, Semenza GL, Van Obberghen E. Insulin stimulates hypoxia-inducible factor 1 through a phosphatidylinositol 3-kinase/targetof rapamycindependent signaling pathway. J Biol Chem. 2002;277(31): 27975-27981.

4. Hudson CC, Liu M, Chiang GG, Otterness DM, Loomis DC, Kaper F, et al. Regulation of hypoxia-inducible factor lalpha expression and function by the mammalian target ofrapamycin. Mol Cell Biol. 2002;22(20):7004-7014

5. Brugarolas JB, Vazquez F, Reddy A, Sellers WR, Kaelin WG Jr. TSC2 regulates VEGF through mTOR-dependent and -independent pathways. Cancer Cell. 2003;4(2):147-158.

6. Thomas GV, Tran C, Mellinghoff IK, Welsbie DS, Chan E, Fueger $B$, et al. Hypoxia-inducible factor determines sensitivity to inhibitors of mTOR in kidney cancer. Nat Med. 2006;12(1):122-127.

7. Atkins MB, Hidalgo M, Stadler WM, Logan TF, Dutcher JP, Hudes GR, et al. Randomized phase II study of multiple dose levels of CCI-779, a novel mammalian target of rapamycin kinase inhibitor, in patients with advanced refractory renal cell carcinoma. J Clin Oncol. 2004;22(5):909-918.

8. Hudes G, Carducci M, Tomczak P, Dutcher J, Figlin R, Kapoor A, et al. Temsirolimus, interferon alpha, or both for advanced renal-cell carcinoma. N Engl J Med. 2007;356(22):2271-2281.

9. Amato RJ, Misellati A, Khan M, et al. A phase II trial of RAD001 in patients (Pts) with metastatic renal cell carcinoma (MRCC). J Clin Oncol 2006; 24:18s (abstract 4530).[Asco]

10. Motzer RJ, Escudier B, Oudard S et al. Efficacy of everolimus in advanced renal cell carcinoma: a double-blind, randomised, placebo -controlled phase III trial. Lancet 2008;372(9637):449456.

11. Ohm JE, Gabrilovich DI, Sempowski GD, et al: VEGF inhibits T-cell development and may contribute to tumor-induced immune suppression. Blood 2003;101:4878-4886.

12. Gabrilovich DI, Ishida T, Nadaf S, et al: Antibodies to vascular endothelial growth factor enhance the efficacy of cancer immunotherapy by improving endogenous dendritic cell function. Clin Cancer Res 1999;(5):2963-2970.
13. Fox SB, Turley H, Cheale M, et al: Phosphorylated KDR is expressed in the neoplastic and stromal elements of human renal tumours and shuttles from cell membrane to nucleus. J Pathol 2004; 202(3):313-320.

14. Yang JC, Haworth L, Sherry RM, et al: A randomized trial of bevacizumab, an anti-vascular endothelial growth factor antibody, for metastatic renal cancer. N Engl J Med 2003;349 (5):427-434.

15. Escudier B, Pluzanska A, Koralewski P for the AVOREN trial investigators. Bevacizumab plus interferon alfa-2a for the treatment of metastatic renal cell carcinoma: a randomized, double-blind phase III trial. Lancet 2007;370(9605):2103-2111.

16. Rini BI, Halabi S, Rosenberg JE et al. CALGB 90206: A phase III trial of bevacizumab plus interferon alpha versus interferon alpha monotherapy in metastatic renal cell carcinoma. ASCO GU Meeting Proceedings 2008: Abs 350 [2008 Genitourinary Cancers Symposium.

17. Motzer RJ, Rini BI, Bukowski RM, et al: Sunitinib in patients with metastatic renal cell carcinoma. JAMA 2006;295(21): 2516-2524.

18. Figlin RA, Hutson TE, Tomcak P et al. Overall survival with sunitinib versus interferon-alfa as first-line treatment of metastatic renal cell carcinoma. ASCO Annual Meeting Proceedings. J Clin Oncol 26: 2008 (May 20 suppl; abstr 5024) [ASCO]

19. Rini B, Rixe R, Bukowski R, et al: AG-013736, a multi-target tyrosine kinase receptor inhibitor, demonstrates anti-tumor activity in a phase 2 study of cytokine-refractory, metastatic renal cell carcinoma (RCC). J Clin Oncol 26: 2008 (May 20 suppl; abstr 4509). [ASCO]

20. Rixe O, Bukowski RM, Michaelson MD, Wilding G, Hudes GR, Bolte O, Motzer RJ, Bycott P, Liau KF, Freddo J, Trask PC, Kim $\mathrm{S}$, Rini BI. Axitinib treatment in patients with cytokine-refractory metastatic renal-cell cancer: a phase II study. Lancet Oncol. 2007;8(11):975-984.

21. Motzer RJ, Hutson TE, Tomczak P, et al: Sunitinib versus interferon alfa in metastatic renal-cell carcinoma. N Engl J Med 2007;356(2):115-124.

22. Hood JD, Bednarski M, Frausto R, et al: Tumor regression by targeted gene delivery to the neovasculature. Science 2002;296(5577):2404-2407.

23. Escudier B, Eisen T, Stadler WM, et al: Sorafenib in advanced clear-cell renal-cell carcinoma. N Engl J Med 2007; 356:125-134.

24. Figlin RA, Hutson TE, Tomcak P et al. Overall survival with sunitinib versus interferon-alfa as first-line treatment of metastatic renal cell carcinoma. J Clin Oncol 26: 2008 (May 20 suppl; abstr 5024). [ASCO]

25. Szczylik C, Demkow T, Staehler M, et al. Randomized phase II trial of first-time treatment with sorafenib versus interferon in patients with advanced renal cell carcinoma: Final results. J Clin Oncol 26: 2008 (May 20 suppl; abstr 5025). [ASCO]

26. Gunaratnam L, Morley M, Franovic A, de Paulsen N, Mekhail $\mathrm{K}$, Parolin DA, et al. Hypoxia inducible factor activates the transforming growth factor-alpha/epidermal growth factor receptor growth stimulatory pathway in VHL(_/) renal cell carcinoma cells. J Biol Chem. 2003;278(45):44966-44974.

27. de Paulsen N, Brychzy A, Fournier MC, Klausner RD, Gnarra JR, Pause A, et al. Role of transforming growth factor-alpha in von Hippel-Lindau (VHL)(_/_) clear cell renal carcinoma cell proliferation: A possible mechanism coupling VHL tumor suppressor inactivation and tumorigenesis. Proc Natl Acad Sci U S A. 2001;98(4):1387-1392.

28. Knebelmann B, Ananth S, Cohen HT, Sukhatme VP. Transforming growth factor alpha is a target for the von HippelLindau tumor suppressor. Cancer Res. 1998;58(2):226-231. 
29. Smith K, Gunaratnam L, Morley M, Franovic A, Mekhail K, Lee S. Silencing of epidermal growth factor receptor suppresses hypoxia-inducible factor-2-driven VHL_/_ renal cancer. Cancer Res. 2005;65(12):5221-5230.

30. Prewett M, Rothman M, Waksal H, et al: Mouse-human chimeric antiepidermal growth factor receptor antibody C225 inhibits the growth of human renal cell carcinoma xenografts in nude mice. Clin Cancer Res. 1998; 4:2957-2966.

31. Rowinsky EK, Schwartz GH, Gollob JA, Thompson JA, Vogelzang NJ, Figlin R, et al. Safety, pharmacokinetics, and activity of ABX-EGF, a fully human anti-epidermal growth factor receptor monoclonal antibody in patients with metastatic renal cell cancer. J Clin Oncol. 2004;22(15):3003-3015.

32. Motzer RJ, Amato R, Todd M, Hwu WJ, Cohen R, Baselga J, et al. Phase II trial of antiepidermal growth factor receptor antibody C225 in patients with advanced renal cell carcinoma. Invest New Drugs. 2003;21(1):99-101.
33. Hainsworth JD, Sosman JA, Spigel DR, Edwards DL, Baughman C, Greco A. Treatment of metastatic renal cell carcinoma with a combination of bevacizumab and erlotinib. J Clin Oncol. 2005;23(31):7889-7896.

34. Bukowski RM, Kabbinavar FF, Figlin RA, Flaherty K, Srinivas $\mathrm{S}$, Vaishampayan U, et al. Randomized phase II study of erlotinib combined with bevacizumab compared with bevacizumab alone in metastatic renal cell cancer. J Clin Oncol. 2007;25(29):4536-4541.

Correspondencia autor: Dr. Joaquim Bellmunt Servicio de Oncologia Médica. Hospital del Mar,

Paseo Maritimo 25-29- Barcelona 08003. Tel: +34 932483137

E-mail autor: jbellmunt@imas.imim.es

Información artículo: Original

Trabajo recibido: marzo 2009

Trabajo aceptado: abril 2009 